\title{
Ultra-broadband ptychography with self-consistent coherence estimation from a high harmonic source
}

\author{
M. Odstrcil ${ }^{a, b}$, P.Baksh ${ }^{a}$, H.Kim ${ }^{a, b}$, S. A. Boden ${ }^{c}$, W. S. Brocklesby ${ }^{a}$, J. G. Frey ${ }^{d}$ \\ ${ }^{a}$ Optoelectronics Research Centre, University of Southampton, SO17 1BJ, UK \\ ${ }^{b}$ RWTH Aachen University, Experimental Physics of EUV, JARA-FIT, Steinbachstrasse 15, \\ 52074, Aachen, Germany \\ ${ }^{c}$ Faculty of Physical Sciences and Engineering, University of Southampton, Southampton, \\ SO17 1BJ, UK \\ ${ }^{d}$ Chemistry, Faculty of Natural and Environmental Sciences, University of Southampton, \\ Southampton, SO17 1BJ, UK
}

\begin{abstract}
With the aim of improving imaging using table-top extreme ultraviolet sources, we demonstrate coherent diffraction imaging $(\mathrm{CDI})$ with relative bandwidth of $\approx 20 \%$. The coherence properties of the illumination probe are identified using the same imaging setup. The presented methods allows for the use of fewer monochromating optics, obtaining higher flux at the sample and thus reach higher resolution or shorter exposure time. This is important in the case of ptychography when a large number of diffraction patterns need to be collected. Our microscopy setup was tested on a reconstruction of an extended sample to show the quality of the reconstruction. We show that high harmonic generation based EUV tabletop microscope can provide reconstruction of samples with a large field of view and high resolution without additional prior knowledge about the sample or illumination.
\end{abstract}

Keywords: ptychography, CDI, HHG, broadband, table-top, EUV, lensless

\section{INTRODUCTION}

Coherent diffraction imaging (CDI) methods use the intensity from a measured diffraction pattern in combination with a priori knowledge to numerically reconstruct both attenuation and relative phase shift introduced by the diffracting specimen. CDI was firstly introduced in the X-ray synchrotron community ${ }^{1}$ as a coherent diffraction based microscopy method for aperiodic samples using prior knowledge of compactness of the sample. ${ }^{2,3}$ In the last decade, a more modern and powerful CDI method for reconstruction of extended samples called ptychography was introduced. ${ }^{4,5}$ The ptychography method is based on the idea of illuminating multiple overlapping regions with a structured illumination. The prior knowledge used in this method is that the illumination and the sample are constant during the scan and that the scanning positions are precisely known. This additional knowledge allows for the reconstruction of the complex illumination and object function with a suitable numerical algorithm.

CDI methods are crucial for hard X-ray imaging when the attenuation of most materials is low and majority of the interaction between material and photons is caused by elastic scattering. This interaction results in low contrast in standard lens-based bright-field microscopy because most of the information is stored in the phase shift of the exit wave. Further to this, X-ray optics required to collect scattered light are challenging to manufacture, expensive, and experience high losses. However, CDI is not limited to hard X-rays and can be generally used in any spectral range or also for coherent electron beam..$^{2,3}$

One of the main drawbacks of CDI methods are strict requirements on overall beam quality: high spatial and temporal coherence, related to the oversampling ratio of the diffraction pattern, ${ }^{6}$ and good temporal stability. These requirements of high brightness, short wavelength coherent illumination leads to increased interest in tabletop coherent EUV sources. One of the most significant tabletop sources in EUV range uses a high harmonic

Correspondence author: M. Odstrcil, E-mail: mo1e13@soton.ac.uk 
generation (HHG) process that can provide sufficient flux for imaging down to $13 \mathrm{~nm}$ wavelength. ${ }^{7}$ The HHG process uses a high intensity laser beam to drive a strongly non-linear process, emitting radiation at odd integer multiples of the driving laser field, while conserving much of the coherence of the fundamental laser beam.

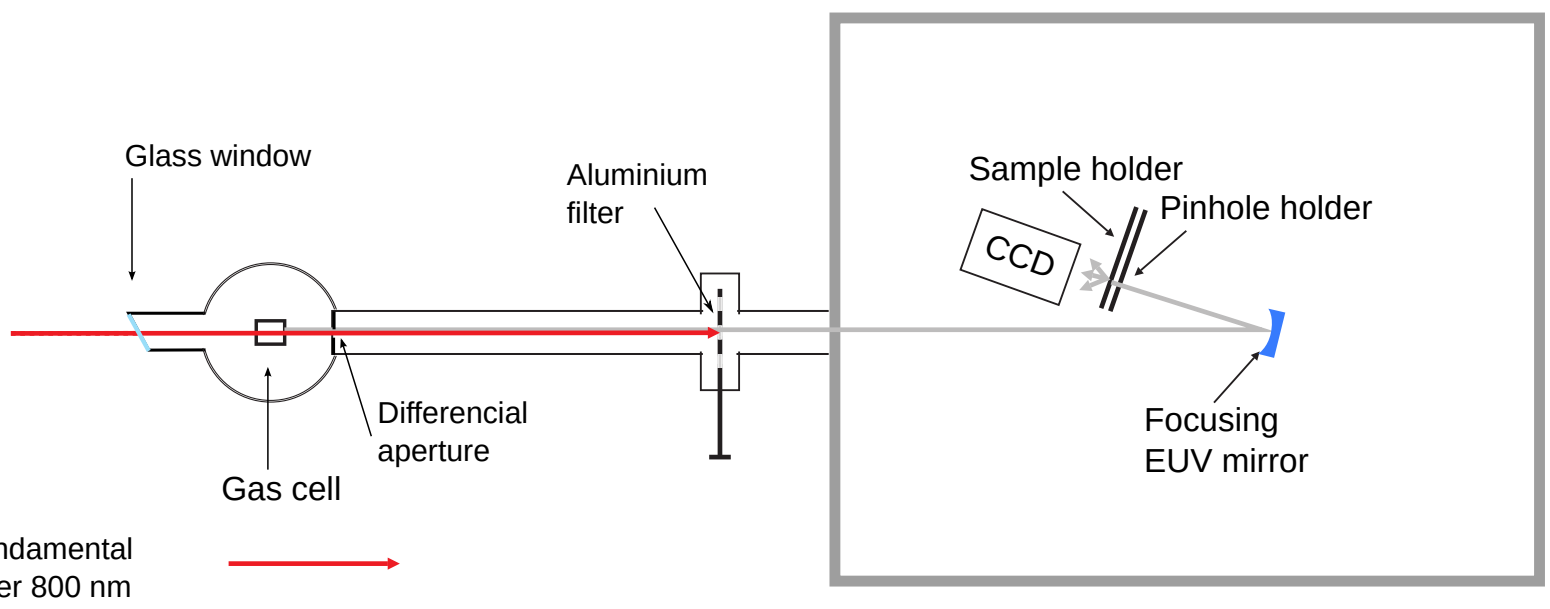

laser $800 \mathrm{~nm}$

EUV radiation

$(\sim 28 \mathrm{~nm})$

Figure 1. A schema of the experimental setup. EUV light is produced in a gas cell by interaction between driving laser at $800 \mathrm{~nm}$ and 80 mbar argon gas. The IR radiation is removed by an aluminum foil and EUV light is focused onto the sample and where a diffraction pattern is recorded on an EUV camera

\section{EXPERIMENT}

Our setup is shown in the Fig 1. The $800 \mathrm{~nm}$ ultrafast laser beam of pulse length $40 \mathrm{fs}$ and an average power $1.8 \mathrm{~W}$ is focused into a $3 \mathrm{~mm}$ long gas-cell using a $75 \mathrm{~cm}$ focal length lens. The gas-cell contains 80 mbar of argon gas and this is where the high harmonic radiation is generated. The background pressure in the generation chamber is $1.5 \times 10^{-1}$ mbar. The input and output apertures of the gas cell are laser drilled in order to limit attenuation of the generated EUV beam by residual argon gas. Moreover, small differential pumping apertures are used to separate the generation chamber from the imaging chamber (see Fig 1). The EUV and IR beam are separated by a single $200 \mathrm{~nm}$ thick aluminum filter. The distance between the gas cell and the aluminum filter is $1.25 \mathrm{~m}$, in order to prevent damage of the filter by the high intensity driving beam.

The EUV beam is focused using a single spherical $\mathrm{B}_{4} \mathrm{C} / \mathrm{Si}$ multilayer mirror with focal length of $20 \mathrm{~cm}$. The folding angle of the focusing mirror, which is limited by size of the camera, was estimated to be $7^{\circ}$. This leads to a significant astigmatism of the beam (see Fig 2). The illumination incident on the sample is defined by a $7 \mu \mathrm{m}$ pinhole. The diffracted light is collected by a CCD camera (Andor DX-434 $(1024 \times 1024 \mathrm{px}))$. The sample is mounted on a 3-dimensional nanoprecision piezo stage (Smaract - SLC-1730). The sample stage and pinhole are mounted on a second shared 3-dimensional stage in order to minimize sample vibrations and still allow precise adjustment of the pinhole position into the center of the focused EUV beam.

EUV beam stability is a significant issue for table top experiments such as high harmonic generation (HHG). Harmonic generation processes conserve the pointing stability of the driving laser while the beam divergence is roughly reduced by the square root of the harmonic order. ${ }^{8}$ The consequence is that the ratio between beam vibrations and beam size is increased and the produced EUV beam can move a significant fraction of beam radius. $^{9}$

The EUV beam was spectrally filtered by a single $\mathrm{B}_{4} \mathrm{C} / \mathrm{Si}$ multilayer mirror with peak reflexivity around $30 \mathrm{~nm}$ wavelength. Using only a single mirror provides up to 5 times more signal ${ }^{10}$ however the it results in broadband EUV illumination caused by imperfect monochromatization. The precise bandwidth depends on the spectral characteristics of the mirror and also on other parameters that are varied at the source such as 


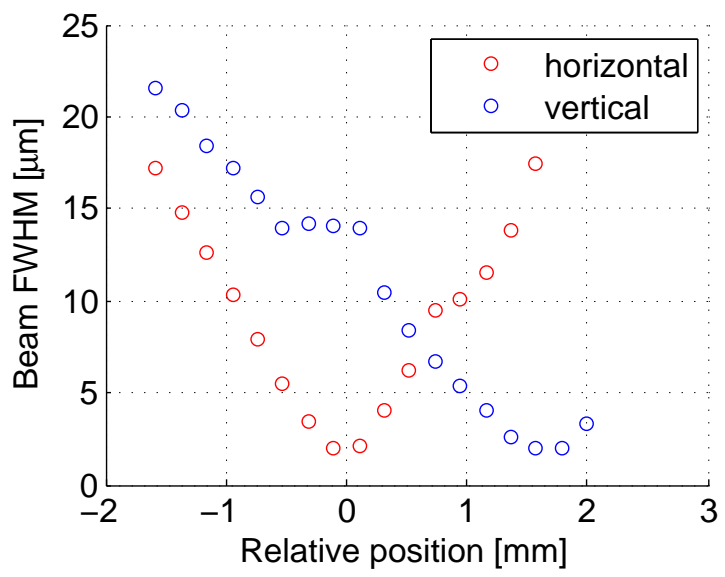

Figure 2. Full width in half maximum (FWHM) of the EUV beam measured through focus. The beam was measured by a 2 microns diameter pinhole scan, therefore the measured size of the beam is convolved with a 2 microns aperture. The significant astigmatism was caused by the folding angle $7^{\circ}$ of the focusing EUV mirror. The FWHM in the circle of least confusion $(\mathrm{CLF})$ is around $7 \mu \mathrm{m}$.

input laser power or phase matching of the HHG source. Because these parameters can vary between different experiments, it is advantageous to be able to precisely measure the spectrum before each experiment. In order to avoid changes of the imaging setup, we have used spectral characterization of the beam by analysis of the Youngs slits diffraction pattern. ${ }^{11}$ Known parameters of the slits e.g. width $b$, separation $d$ and distance from camera $Z$ can be used to calculate Young's slits patterns for the selected wavelength $\lambda$

$$
I(x) \propto\left[1+\gamma \cos ^{2}\left(\frac{\pi \mu x}{Z \lambda}\right)\right] \operatorname{sinc}^{2}\left(\frac{\pi b x}{Z \lambda}\right)
$$

Because the measured broadband diffraction pattern is an incoherent sum of multiple wavelengths, it can be written as a system of linear equations that can be solved by the Tikhonov regularization. ${ }^{12}$ The resulting spectrum is shown in the Fig 3. The diffraction-limited resolution of our Young's slits spectrometer is $0.5 \mathrm{~nm}$.

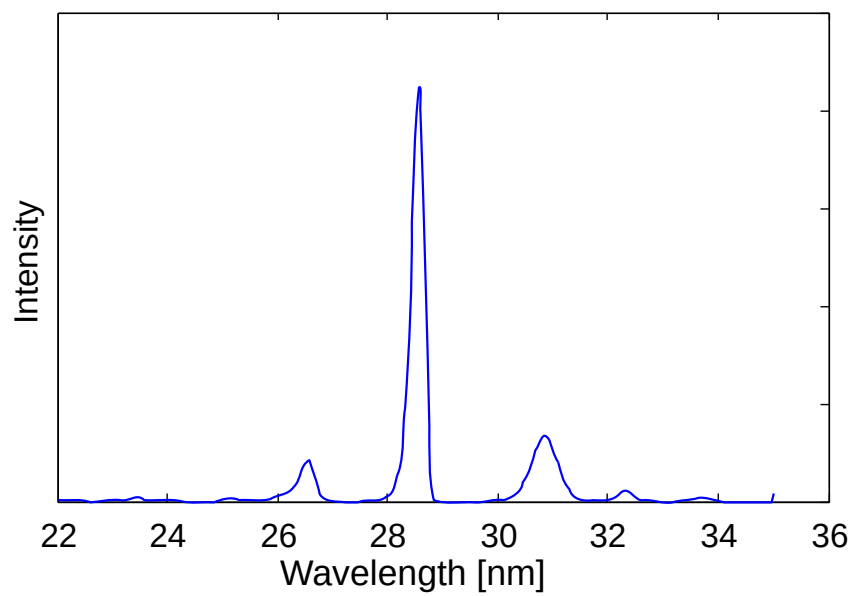

Figure 3. Reconstruction of broadband spectrum that was used to illuminate our sample. A single main peak surrounded by two weaker satellite peaks. The diffraction-limited resolution of the Youngs slits spectrometer is $0.5 \mathrm{~nm}$.

The measured spectrum in the Fig. 3 can be used to calculate the spectral bandwidth. We have used the weighted standard deviation of the spectrum centered at wavelength $\hat{\lambda}$

$$
\frac{\delta \lambda}{\hat{\lambda}}=\frac{1}{\hat{\lambda}} \sqrt{\frac{\sum S(\lambda-\hat{\lambda})^{2}}{\sum S}}
$$




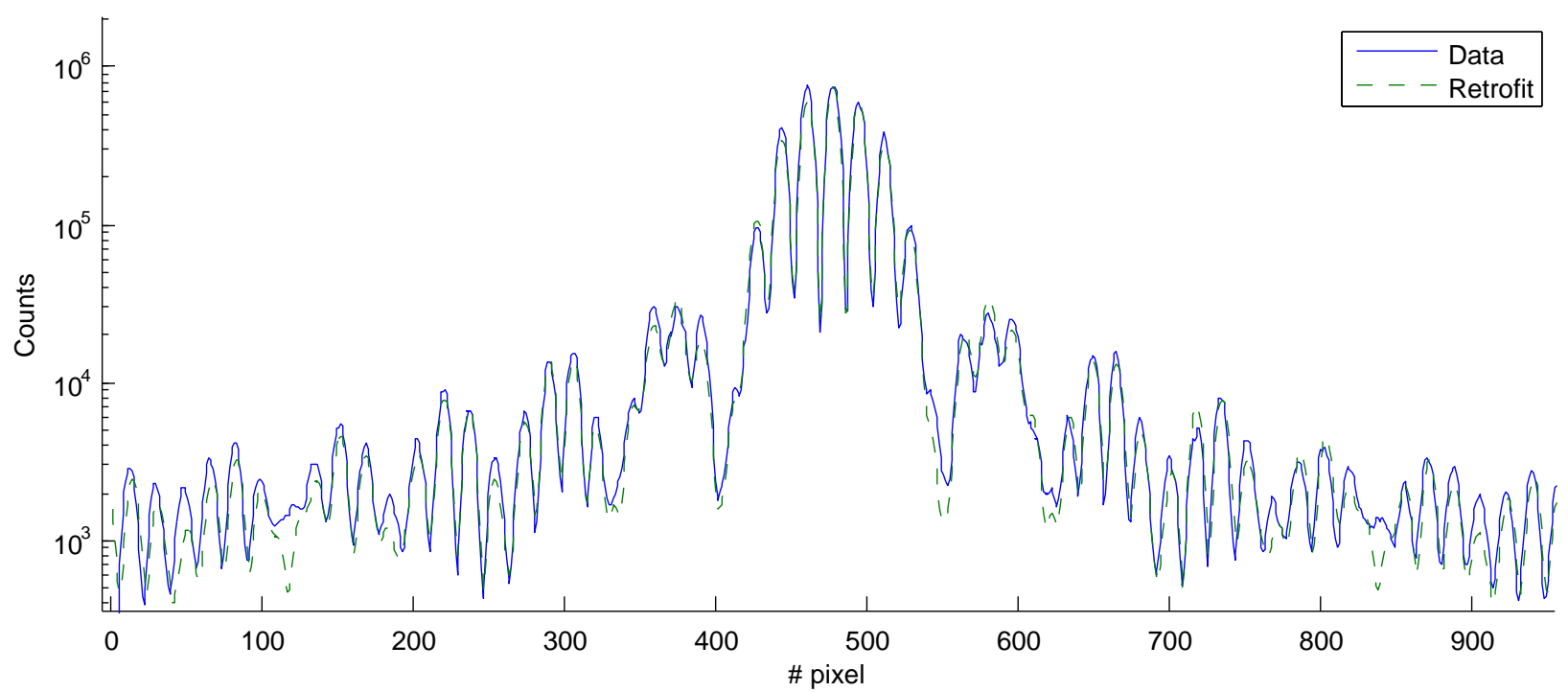

Figure 4. Intensity profile of measured diffraction data in log scale and retrofit from an incoherent sum of monochromatic patterns.

which results into effective bandwidth $20 \%$. The finest spatial frequency $d_{\min }$ that can be observed in the diffraction pattern without incoherence effects ${ }^{6}$ should be equal to

$$
d_{\min }=\frac{\delta \lambda}{\lambda} \frac{W}{2}
$$

where $W$ denotes the size of the illumination. Using a $7 \mu \mathrm{m}$ pinhole, this theoretically limits the achievable CDI resolution to mere $0.7 \mu \mathrm{m}$.

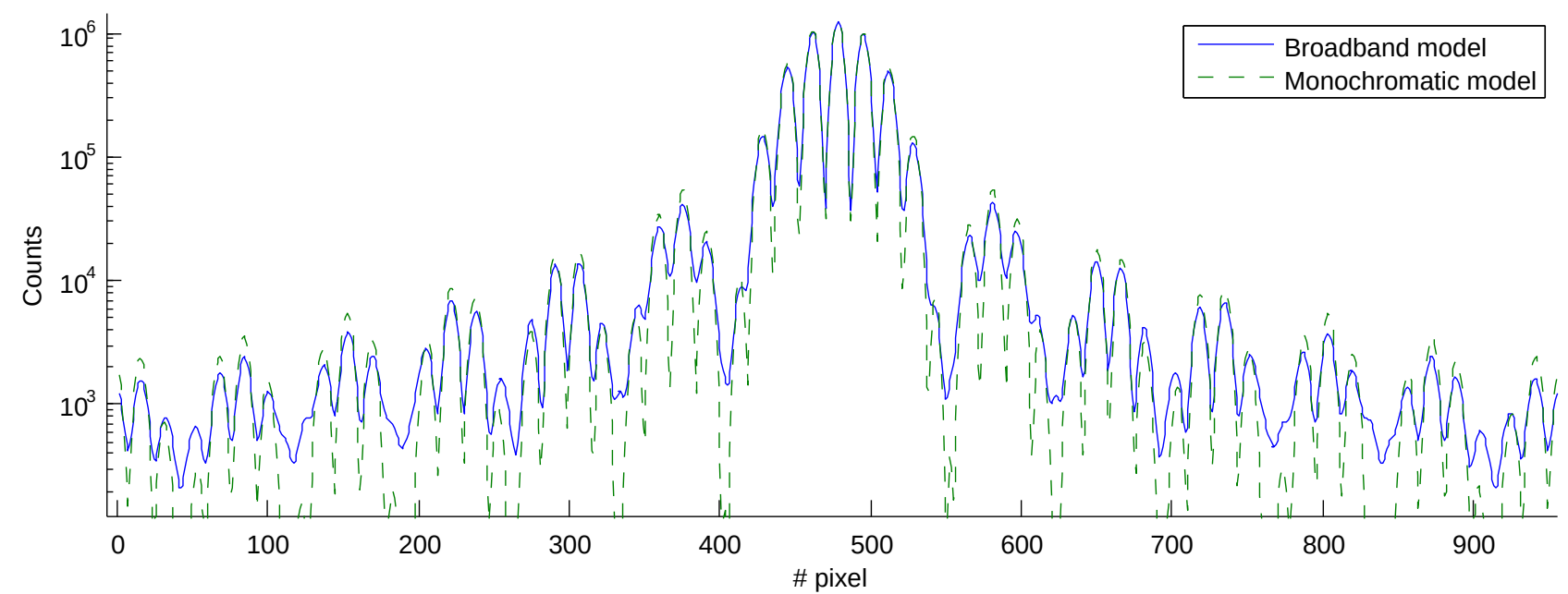

Figure 5. Comparison between monochromatic (dashed line) and broadband (full line) model of the Young slits diffraction pattern.

The effect of the limited coherence in reducing the visibility of the diffraction fringes is shown in Fig 5 . Despite limited coherence, the fringe visibility stays almost constant over the whole diffraction pattern due to very narrow spectral lines produced by the HHG process,

Another important parameter for imaging applications is the lateral coherence or visibility $V$ of the diffraction pattern $I$

$$
V=\frac{I_{\max }-I_{\min }}{I_{\max }+I_{\min }}
$$


High harmonic generation produces EUV light with lateral coherence length comparable with the beam diameter. The main effect reducing the fringes visibility in our setup is limited pointing stability of the EUV beam caused mainly by movements of the driving IR laser. Figure 6 shows the dependence of the Young's double slits pattern visibility on slit separation. This allows to estimate an equivalent of spatial coherence length to $10 \mathrm{microns}^{13}$ this roughly corresponds to our beam size as observed in Ref. 14.

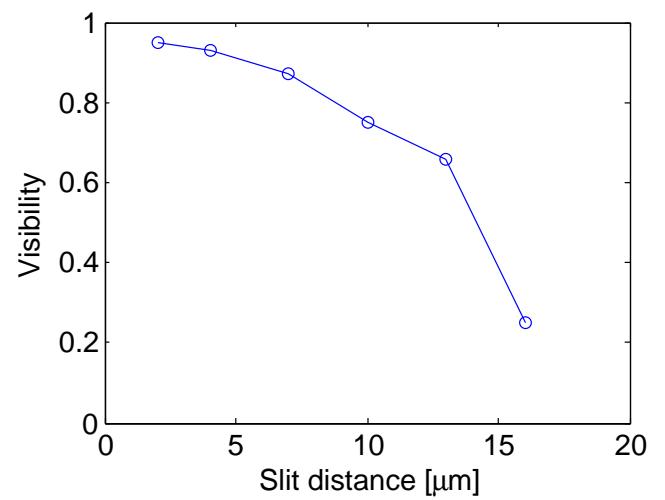

Figure 6. Visibility of the Youngs doubles slits pattern for different separation of slits 2,4,7,10,13 microns. The slits were placed in the circle of least confusion (see Fig 2). The equivalent of the lateral coherence length can be roughly estimated to 8 microns $^{13}$

Because the visibility over longer exposure times is mostly limited by the pointing stability of the driving laser, in our setup, we have attempted to solve this issue by designing our own PID based stabilization system. The beam is moved by four actuators with precision better than $0.2 \mu \mathrm{rad}$ and measured by two CMOS cameras. The stabilization rate is up to $20 \mathrm{~Hz}$. This is sufficient for correction of drifts caused by cryo-system in the laser amplifier and other thermal drifts. Faster fluctuations were suppressed mechanically by perfect sealing of the laser beam path and removing all other possible sources of sound vibrations such as vacuum pumps and chillers. The time evolution of the beam pointing stability at the gas-cell position with and without stabilization is shown in the Fig 7.

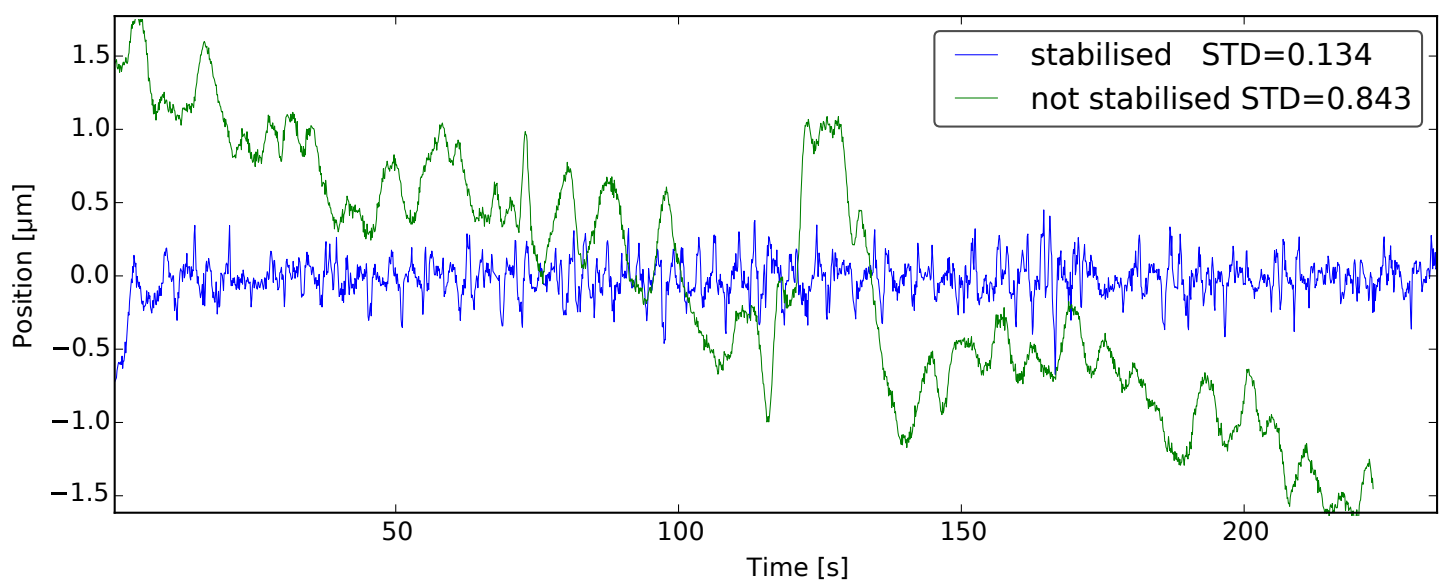

Figure 7. Pointing stability of the driving laser over $220 \mathrm{~s}$ period with and without stabilization.

\section{CDI EXPERIMENT}

An aperiodic grid covered by a $100 \mathrm{~nm}$ layer of gold was used as our initial sample. Aperiodicity and high contrast significantly improve convergence in the case of an imperfect initial estimate of the imaging setup parameters. The sample was illuminated through a 7 micron diameter circular aperture placed in the CLC. We have collected 578 high dynamic range diffraction patterns with maximally $0.5 \mathrm{~s}$ exposure time and $62.6 \mathrm{kHz}$ pixel readout 
speed at the camera. The distance between the sample and camera was estimated to $25 \mathrm{~mm}$ resulting in a diffraction limited resolution of $60 \mathrm{~nm}$. Collected diffraction patterns from our sample were reconstructed using the extended ptychography engine (ePIE). ${ }^{5,15}$ Ptychography allows recovery of attenuation and relative phase shift introduced by the object $O(\mathbf{r})$ and also the complex illumination probe $P(\mathbf{r})$ that is shifted by a know distance $R_{i}$ into different regions of the sample. The solution is found by alternating the real space and the Fourier space constraints. The real space constraint is enforced by the overlap of adjacent scanning regions and the Fourier space constraint is defined by the measured data. The details of the ePIE method is described in Ref.15. Because our illumination is broadband, we have used the polyCDI method ${ }^{16}$ in combination with the spectrum reconstructed using the Young's double slits pattern (Fig 3) that was measured before the experiment. Note that the assumptions of the polyCDI method (e.g. flat illumination wavefront) are not fulfilled, however it is still a very good approximation for our case. The fast pointing instability of the beam was approximated by Gaussian blur of the diffraction pattern, and a self-consistent relaxation method described in Ref 17 was applied.

The resulting reconstruction is shown in Fig. 8a). The reconstruction is showing roughly $25 \times 25 \mu \mathrm{m}$ region of our sample. The reconstruction consists of fully empty (green) and non-transparent (black) regions. The sample was expected to be binary because of the gold layer deposited on the surface. The reconstruction compares very well with the transmission electron microscope image Fig. 8b). The reconstructed illumination probe is shown in left corner of the Fig 8a). The illumination function is result of the curved EUV beam cropped by the $7 \mu \mathrm{m}$ pinhole and propagated roughly $55 \mu \mathrm{m}$ on sample plane.

Despite the very good reconstruction quality, the reconstruction still shows artefacts in the low spatial frequencies. The artefacts are clearly visible along the sharp high contrast edges of our sample. These artefacts can be caused by a large number of systematic errors, for example. The artifacts in the low spatial frequencies are probably caused partly by remaining limited stability of the beam, imperfect stitching of multiple exposures in the high dynamic range image or because the sample to pinhole distance was slightly varying and the sample was not in focus over all the scanned region. Beam stability issues could be reduced if a smaller illumination pinhole is used; however, this would lead into a smaller field of view. Reduction of the dynamic range is possible by more curved illumination on the pinhole (e.g. by a shorter focal length mirror), however it would be technically challenging due to high folding angle in our setup. Another possible cause of low frequency errors is the nonplanar structure of our gold covered sample that could result in reflections interfering with the transmitted illumination wave.

\section{CONCLUSION}

We have presented initial results from a broadband ptychography setup in the transmission geometry using a high harmonic source at $29 \mathrm{~nm}$ wavelength. We have reconstructed a high contrast sample, however generally any EUV transparent sample can be imaged. We have reached diffraction limited resolution $60 \mathrm{~nm}$ using $0.52 \mathrm{~s}$ exposure time and large field of view of $25 \times \mu \mathrm{m}$. This was made possible due to higher photon flux available because the beam was spectrally filtered only by a single multilayer mirror and absorption edge of argon gas. Spatial filtering of the EUV beam was done by a $7 \mu \mathrm{m}$ diameter pinhole while the beam FWHM was also around $7 \mu \mathrm{m}$. We have tested that the coherence properties of the HHG beam can be identified directly with the same imaging setup that was used for ptychography. This eliminates the need for additional equipment and thus simplify the HHG microscope design.

\section{ACKNOWLEDGMENT}

M.O. and H.K. acknowledge financial support from the EU FP7 Erasmus Mundus Joint Doctorate Programme EXTATIC under framework partnership agreement FPA-2012-0033.

\section{REFERENCES}

[1] Miao, J., Charalambous, P., Kirz, J., and Sayre, D., "Extending the methodology of x-ray crystallography to allow imaging of micrometre-sized non-crystalline specimens," Nature 400(6742), 342-344 (1999).

[2] Nugent, K. A., "Coherent methods in the x-ray sciences," Advances in Physics 59(1), 1-99 (2010). 


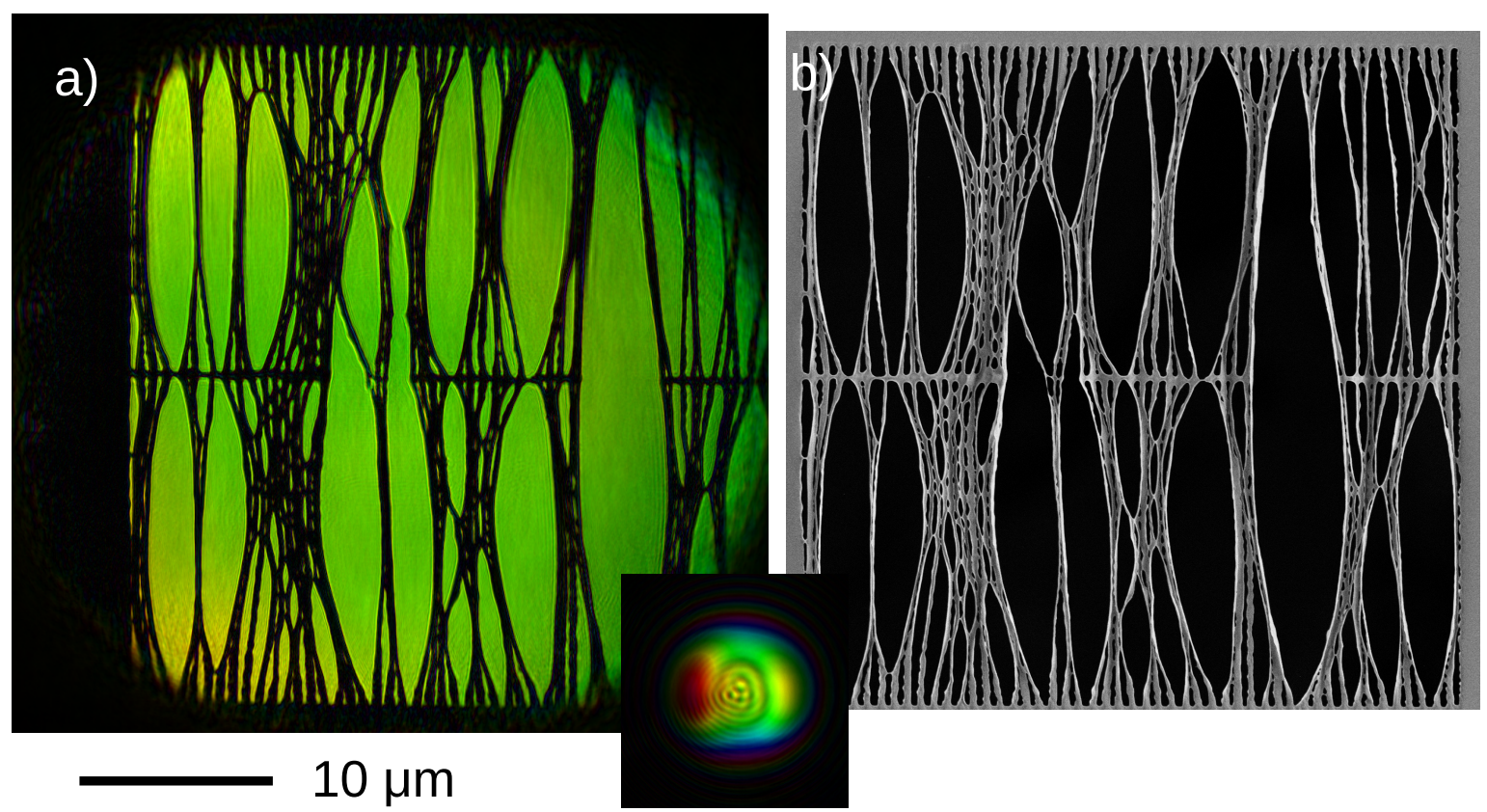

Figure 8. (a) Reconstruction of an aperiodic grid sample using the ptychography method using the hue-saturation-value color scheme to show amplitude and phase of the reconstructed electric field distribution. (b) Image of the sample from an electron microscope. Both sample images and pinhole are shown at the same scale.

[3] Miao, J., Sandberg, R. L., and Song, C., "Coherent x-ray diffraction imaging," Selected Topics in Quantum Electronics, IEEE Journal of 18(1), 399-410 (2012).

[4] Thibault, P. and Elser, V., "X-ray diffraction microscopy," Condensed Matter Physics 1 (2010).

[5] Rodenburg, J., "Ptychography and related diffractive imaging methods," Advances in Imaging and Electron Physics 150, 87-184 (2008).

[6] Spence, J., Weierstall, U., and Howells, M., "Coherence and sampling requirements for diffractive imaging," Ultramicroscopy 101(2), 149-152 (2004).

[7] Seaberg, M. D., Adams, D. E., Townsend, E. L., Raymondson, D. A., Schlotter, W. F., Liu, Y., Menoni, C. S., Rong, L., Chen, C.-C., Miao, J., et al., "Ultrahigh $22 \mathrm{~nm}$ resolution coherent diffractive imaging using a desktop $13 \mathrm{~nm}$ high harmonic source," Optics express 19(23), 22470-22479 (2011).

[8] New, G., [Introduction to Nonlinear Optics], Cambridge University Press (2011).

[9] Erny, C., Mansten, E., Gisselbrecht, M., Schwenke, J., Rakowski, R., He, X., Gaarde, M., Werin, S., and L'Huillier, A., "Metrology of high-order harmonics for free-electron laser seeding," New Journal of Physics 13(7), 073035 (2011).

[10] Parsons, A., Chapman, R., Baksh, P., Mills, B., Bajt, S., Brocklesby, W., and Frey, J., "Ultra-broadband support determination for extreme ultraviolet coherent diffractive imaging from a high harmonic source," Journal of Optics 15(9), 094009 (2013).

[11] Dilanian, R. a., Chen, B., Teichmann, S., Dao, L. V., Quiney, H. M., and Nugent, K. a., "High-harmonicgeneration spectrum reconstruction from Young's double-slits interference pattern using the maximum entropy method," Optics Letters 33, 2341 (Oct. 2008).

[12] Tikhonov, A., "Solution of incorrectly formulated problems and the regularization method," in [Soviet Math. Dokl.], 5, 1035-1038 (1963).

[13] Attwood, D., [Soft x-rays and extreme ultraviolet radiation: principles and applications], Cambridge university press (1999).

[14] Bartels, R. A., Paul, A., Green, H., et al., "Generation of spatially coherent light at extreme ultraviolet wavelengths," Science 297(5580), 376-378 (2002).

[15] Maiden, A. M. and Rodenburg, J. M., "An improved ptychographical phase retrieval algorithm for diffractive imaging," Ultramicroscopy 109(10), 1256-1262 (2009). 
[16] Abbey, B., Whitehead, L. W., Quiney, H. M., Vine, D. J., Cadenazzi, G. A., Henderson, C. A., Nugent, K. A., Balaur, E., Putkunz, C. T., Peele, A. G., et al., "Lensless imaging using broadband x-ray sources," Nature Photonics 5(7), 420-424 (2011).

[17] Clark, J. N. and Peele, A. G., "Simultaneous sample and spatial coherence characterisation using diffractive imaging," Applied Physics Letters 99(15), 154103 (2011). 